\title{
The first pictures: perceptual foundations of Paleolithic art
}

\author{
John Halverson \\ Stevenson College, University of California, Santa Cruz, CA 95064, USA \\ Received 17 April 1990, in revised form 18 July 1991
}

Abstract. Paleolithic representational art has a number of consistent characteristics: the subjects are almost always animals, depicted without scenic background, usually in profile, and mostly in outline; the means of representation are extremely economical, often consisting of only a few strokes that indicate the salient features of the animal which are sufficient to suggest the whole form; and it is naturalistic to a degree, but lacks anything like photographic realism. Two elementary questions are raised in this essay: (i) why did the earliest known attempts at depiction have just these characteristics and not others? and (ii) how are objects so minimally represented recognizable? The answers seem to lie with certain fundamental features of visual perception, especially figure-ground distinction, Gestalt principles of closure and good continuation, line surrogacy, component feature analysis, and canonical imaging. In the earliest pictures the graphic means used are such that they evoke the same visual responses as those involved in the perception of real-world forms, but eschew redundancies of color, texture, linear perspective, and completeness of representation.

\section{Paleolithic art}

Although there is a substantial amount of sculpture from the Paleolithic era and many quasi-geometrical designs, most Paleolithic art is pictorial, consisting of many thousands of recognizable engravings, drawings, and paintings. It is these I am concerned with here. An examination of these earliest pictures from the standpoint of perceptual psychology may be useful in understanding why they have the particular characteristics they have.

Paleolithic art is notoriously difficult to date (Bahn and Vertut 1988), but there is a firm consensus among prehistorians that the great bulk of it was produced in the Solutreo-Magdalenian period approximately 20000 to 10000 years ago, with a rela* tively small number of examples going back through another 10000 years to the Aurignacian period. It was entirely the work of modern Homo sapiens of the CroMagnon type; Neanderthal people apparently produced no figural art of any kind. The great majority of it is concentrated in Western Europe in the Franco-Cantabrian region of southern France and northern Spain, but there are many examples elsewhere in Europe and even in Africa.

Graphic depictions were made on unprepared surfaces of rock or bone by painting, engraving, and carving in low relief. The surfaces used were natural walls, ceilings, and floors of caves and overhang shelters, and small portable pieces of stone and bone. Depictions on stationary surfaces are usually referred to as 'parietal art', being mostly found on the walls of caves; depictions on portable material are referred to as 'mobiliary art'. Frequently, suggestive natural features such as ridges and fissures were incorporated into depictions; a curved ridge, for example, can represent a bison's spine, or a small rock protuberance an animal's eye. It is often assumed that depictions were also made on perishable materials, such as hide, wood, or bark, that have not survived.

Throughout this great temporal and geographical spread, Paleolithic graphic art retained quite a number of very consistent formal and referential characteristics (Leroi-Gourhan 1967, 1982; Sandars 1985; Ucko and Rosenfeld 1967). It is, in the 
first place, overwhelmingly animal art, with only a few human or human-like images, probably no representations of vegetable life, and certainly no landscapes. Animals are never depicted with any kind of background; there is no scenic element in these pictures, only figure and ground, the figure being the animal and the ground being merely the 'other' side of the picture's contour or line, where the rock or bone surface on which the figure is drawn or engraved is left unmodified. Parietal figures are seldom oriented to a consistent horizontal (they may be rotated as much as a full $180^{\circ}$ to appear upside down from the viewer's station orientation), and neighboring figures very frequently ignore realistic size relations (an ibex may be as large as an adjacent horse).

With very few exceptions, the animal figures are portrayed in profile, often in 'strict' profile, ie with only two legs showing or one horn, and sometimes (though less commonly) in 'twisted perspective', where the animal's horns are shown from the front on a profiled figure. But with whatever slight variations, profile is the near absolute norm.

Occlusive overlap is fairly frequent in the depiction of the legs of a single animal figure, but otherwise the representation of distal planes by occlusion is rare, and there seem to be no unambiguous attempts at representing distance by diminishing size from foreground to background. There are, in fact, no plausible examples of any kind of linear convergence perspective. 'Superpositioning', where one figure is drawn over another without any regard to visual occlusion, is much more common.

Partial or 'abbreviated' representation occurs very frequently (Lorblanchet 1977): heads without bodies, rarely bodies without heads, a few lines depicting the outline of head and back and sometimes merely the belly and part of the legs; feet are not commonly shown even when the rest of the figure is more-or-less complete.

Above all, Paleolithic art is an art of outline. The fully painted polychrome figures of Altamira and Lascaux are the glories of cave art and naturally the most frequently reproduced and most familiar paintings of the era, but they are exceptional; most of the art-perhaps ninety percent-is simple outline. Engravings especially, which outnumber paintings, are almost all outline figures.

Finally, Paleolithic art is in a certain general sense 'realistic'. It is not in the least photographic, nor is there anything remotely approaching 'trompe l'oeil' in Paleolithic art-in fact, it is closer to cartoon art than to any other modern art form; it is realistic in the elementary sense that it permits fairly easy recognition of the subjects depicted.

\section{Recognition of pictures}

But how is this recognition made? How do scratches or lines of pigment 'represent' anything? Objectively considered, pictures are thoroughly unnatural, especially outline depictions. In nature, our ancestors would have seen reflections in water, but these are not only fairly replete images, they also provide optic information concerning depth and are mobile when they are self or animal reflections, and thus are not very much like flat motionless depicted images. Shadows are two-dimensional but are also mobile and intrinsically and obtrusively connected with the object casting the shadow. Impressions left in soft earth, such as footprints, are essentially outlines and immobile, and therefore somewhat picture-like.

Perhaps the closest visual object in nature to a two-dimensional picture would be a still back-lit figure silhouetted against the sky or other relatively undifferentiated background, such as grassland, especially at a distance greater than $135 \mathrm{~m}$ or so where binocular effects cease (Haber and Hershenson 1980) and the object is in fact optically two-dimensional. In good lighting, a visual object, such as an animal, presents a complex optical array that includes color and texture gradients that help define mass; they provide 'eidolic' cues (Deregowski 1980, 1990) to three-dimensionality. Such cues are totally lacking in the distant silhouette. The only differentiation 
of luminance is between (dark) figure and (light) ground. Since the interior of the figure is undifferentiated, the only distinguishing feature that remains is its external contour, where luminance is sharply discontinuous. Now the silhouette of a rhinoceros, mammoth, or deer, if, as in profile, it displays the animal's distinguishing characteristics of form, is as recognizable as the fully illuminated creature-in some circumstances even more so, as for instance when there is a 'noisy', visually confusing background (of which both natural and man-made camouflage takes advantage). The pictorial outline abstracts from the silhouette its only signifying feature, its occluding edge, or, better, its 'occluding bound' (Kennedy and Silver 1974). Thus, although an unnatural artifact, the pictorial outline successfully exploits a fundamental component of natural object perception.

\subsection{Occlusion and motion parallax}

Occlusion is also essential for distinguishing a figure from its background. In nature, motion, either, of the object or of the viewer, is often necessary for this perception. The efficacy of an animal 'freezing' to escape detection is well known; motion gives it away. Alternatively, even slight head movements of the viewer create motion parallax (the apparent movement of foreground and background objects in opposite directions) that is, shift occlusion as the foreground object successively masks portions of the background. Both kinds of motion confirm that the object occupies a different plane and is therefore a separate entity.

The absence of motion parallax may help account for the reported difficulties of people unfamiliar with photography in comprehending black-and-white still photographs, whereas "motion pictures are almost universally perceived without trouble" (Segall et al 1966, page 33). If a figure-even a familiar person-is photographed against a visually complex background, naive viewers may be unable to discern it, in part because the normal observer motions, such as head movement, that can produce motion parallax in the three-dimensional world and thus aid figure-ground differentiation are ineffectual with two-dimensional photographs, so no figure emerges. On the other hand, when a photograph lacks a competitive background, no problem of comprehension arises. The experience of Forge (1970; cf Deregowski 1980) is instructive. Among the Abelam of New Guinea, he found that even people "pathetically keen" to see pictures of dead relatives had great difficulty discerning the desired image. Even when he drew a heavy line around it, he was not always sure that they really saw it. Evidently he was showing ordinary snapshots, because posed portraits, by contrast, where the subject stood "at attention in front of a white sheet" elicited immediate recognition. In the latter case there is no background, only 'ground', an undifferentiated field, so no confusion is possible, and the absence of motion parallax goes unnoticed.

\subsection{Distinguishing figure from ground}

In artificial visual constructs, such as the familiar Rubin vase, there may be an ambiguity between figure and ground. Even more simply: Is the circle in figure 1 a disc or a hole? The answer in either case depends on equating lines with edges and deciding which space they bound. Either way, the ground is perceived as a continuous underlay. It is very difficult to perceive this or the Rubin figure as simply the juxtaposition on a single plane of two flat cutouts. This may be in part a biological legacy of motion parallax as an accommodation to a three-dimensional real world. Similarly, it is difficult to see figure $2 \mathrm{a}$ as other than two rectangles, one partially occluding the other, even though figure $2 b$ is also a perfectly logical interpretation, as is figure $2 \mathrm{c}$; in fact there are countless logical combinations of lines that will produce the figure. However, human perception did not evolve 'logically' but in response to a three-dimensional world in which occlusion is the norm. 
Returning to figure 1 , we should note that the circle may also be perceived as only a circle, rather than as a boundary of either the space outside or the space inside it (and therefore neither a disc nor a hole). Does this mean the line is not to be equated with a boundary at all? Geometrically yes, but geometrical lines are convenient logical fictions. 'Primitive' perception probably sees the circle as having dimension and thus having its own inner and outer edges with a continuous underlying ground. This becomes evident simply by thickening the line, so that if asked to identify it with a real object, we might call it a hoop or, with more thickening, a tire or a doughnut. And in fact an equivalent physical construction of such a circle, no matter how thin a wire were used, would be three-dimensional with occluding bounds, and would be interpreted as such.

Not all linear contours are occlusive, of course; cracks and corners, for instance, do not imply figure-ground distinction (Kennedy 1974b). Yet it would be difficult to overestimate the importance of this basic component of perception in the real world and in human adaptation.

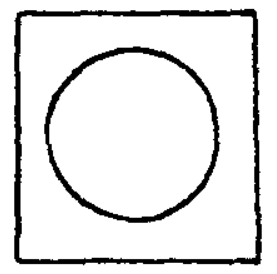

Figure 1. Example of ambiguity between figure and ground.

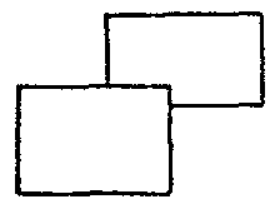

(a)

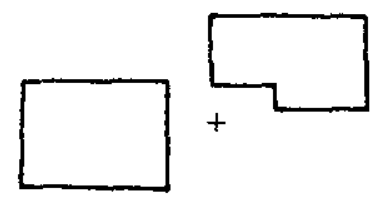

(b)

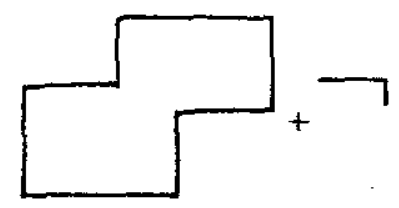

(c)

Figure 2. The figure in (a) is seen as two rectangles, one partially occluding the other, even though (b) and $(c)$ are logical alternative interpretations.

\subsection{Recognition of line drawings}

Of equal importance in the pictorial realm is the capacity of lines to act as 'surrogates' for visual contours (Hochberg 1972). Despite some doubts in the past, there is now strong evidence to suggest that the identification of lines with contours is an innate function of normal human vision (Kennedy 1974b; Kennedy and Silver 1974). This has been shown both developmentally and cross-culturally. Hochberg and Brooks (1962) report that an American child kept away from all pictures until the age of two was able immediately to identify line drawings of familiar objects. Similar results have come from pictureless, or relatively pictureless, tribal societies in Africa and New Guinea. Information from these sources is not always clear-cut because investigators are often experimenting with other problems, especially depth perception-a favorite subject since the work of Hudson (1960)--using outline pictures more-or-less incidentally. But whatever the case with depth perception-and this issue remains controversial-subjects unfamiliar with pictures have no difficulty in identifying objects pictured in outline form (Hudson 1960; Kennedy 1974b; MundyCastle 1966).

The clearest experiment, because solely concerned with the recognition of outline depictions, is that of Kennedy and Ross (1975) with the Songe of New Guinea, where 
they found an extremely high rate of immediate recognition of familiar objects, and most of the subjects who responded incorrectly the first time were, as soon as they were told what the figures represented, quickly able to identify details of the depictions, showing that they did recognize the figures. Deregowski et al (1972; cf Deregowski 1976), on the other hand, found with their subjects, the Me'en of Ethiopia, that although they responded well after the pictures had been printed on familiar material, they were in some cases very slow and in need of coaching. But this may have been, paradoxically, because the drawings used were not bare outlines but richly detailed for texture and shadow; in short, they may have had too much, and therefore potentially distracting, information. If we compare one of Kennedy's figures with one of Deregowski's (figure 3), perhaps we can appreciate how the more cartoon-like cow presented less difficulty to its viewers than the more realistic antelope, and how the latter might be said to contain too much information, because its texturizing tends to obscure definition, and the turned head, though naturalistic, is less easily distinguishable than the profile head of the cow (Kennedy 1974a). (The value of profile representation is suggested by the fact that the picture of a leopard completely in profile that was shown in the Deregowski test received correct responses from almost all subjects.) It should be noted that though cattle were relatively unfamiliar to the Songe, they achieved a recognition rate of $97 \%$, while the dik-dik, a well-known animal to the Me'en, was recognized by only $65 \%$ of the subjects.

Biederman and Ju (1988) have shown that edge-based representations, ie outline drawings, work as well as high-resolution color photographs for object recognition and that they are by themselves sufficient, in most cases, for this purpose; the representation of surface gradients contributes little or nothing.

The universality of outline drawing in both time and space has been shown by Kennedy and Silver (1974), who examined some 657 examples from all over the world from Paleolithic to modern times, and found that $99 \%$ had the common feature of 'occluding bound'. The evidence seems very good, then-and there seems to be none to the contrary-that, in the proper circumstances, object recognition from outline drawings is universal and innate; 'proper circumstances' include such obvious conditions as normal eyesight, some degree of verisimilitude of depiction, and minimal visual noise.

Pictorial perception may not even be a uniquely human ability. There are indications that other animals, especially higher primates, also have the ability, at least potentially (Cabe 1980), so there is some indication of evolutionary continuity, which would support the thesis of innateness.

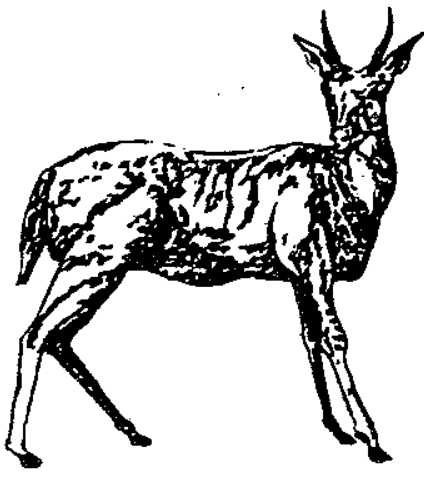

Deregowski's dik-dik

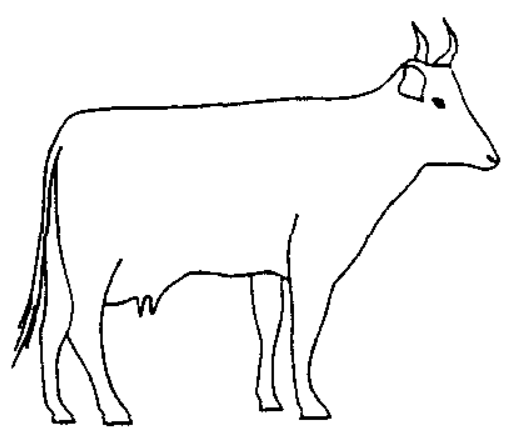

Kennedy's cow

Figure 3. (a) Richly detailed picture of a dik-dik used in the study of Deregowski et al (1972). (b) Outhine drawing of a cow used in the study of Kennedy (1974a). 


\section{Recognition of the figures in Paleolithic art}

Outline depiction is, to a great extent, a matter of simplification, ie the reduction of visual information to the bare necessities. Above all it involves the reduction of complex optical arrays to lines, but the limitation of linear depiction to salient and diagnostic features is also involved. Paleolithic art is thoroughly grounded on the principles of formal simplification and accentuation of salient features, ie those features most useful for identification, such as horns.

Figure 4 shows some fairly typical examples of Paleolithic cave art. They are immediately recognizable as a rhinoceros; an ibex, and a horse, despite extreme simplification (and some distortion). Most obviously, they are in 'strict profile' with only two legs represented and, in the case of the ibex, only one horn. This is visually unrealistic, for it is hardly possible to find a point of observation from which a real animal would appear so. None of the animals has feet. The rhinoceros' larger horn is unnaturally thin and no ear is shown, although the modern rhinoceros' large ears are conspicuous even in profile. The arc of the ibex's horn is unrealistically shallow. All the creatures lack eyes and nostrils, and have no pictorial cues for solidity; in fact they have no insides at all, only, in situ, the quite visibly textured rock surface that is continuous on both sides of the outline. So they are not silhouettes, though they have a silhouette quality, namely the total reliance on form boundary. They are strictly 'epitomic' figures (Deregowski's term) in that they lack any visual depth cues. They are also epitomes in the ordinary sense of being concise representations of classes, in this case animal species. This epitomization is accomplished by selection of distinguishing features to the exclusion of virtually all else. First, overall shape has been adequately captured, ie the relative proportions of the bodies; second, identifying characteristics of each species are shown: the rhinoceros' heavy low-slung head and double horn, the ibex's distinctively curled horn, and the wild horse's typically boxy head and bristly mane. Eyes, nostrils, ears (in two cases), color, and texture are absent, not because they are not different in nature (the rhinoceros' squinty, wrinkled eyes, for instance, are very distinctive), but because they are unnecessary for the pictorial epitome. The depiction of legs is something of a compromise; though not altogether necessary for identification (the animals can still be recognized without legs), it does enhance the sense of completeness. However, the representation of legs is very rudimentary and schematic, mere lines tapering into nothing.
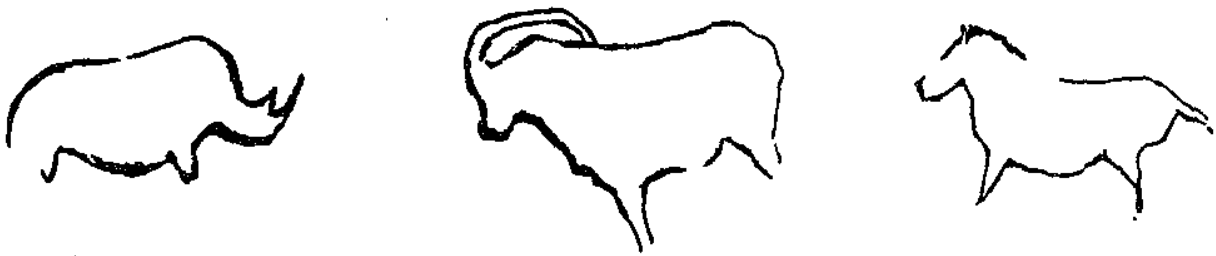

Figure 4. Typical examples of Paleolithic cave art.

\subsection{Role of prior knowledge}

From the earlier discussion of figure and ground, it might be asked what determines figure and ground in these instances of Paleolithic art. The question arises because, unlike drawings on paper, these were, more often than not, made on textured rock surfaces whose own patterns visibly continue right through the outline figures. If the figure were a geometric circle in the same circumstances, we would not be tempted to interpret it as a disc, sphere, or hole; it could only be a hoop (figure 5). Similarly, if the shape were completely alien we would probably attribute the same figure-ground status to it as we would to the circle, ie an empty outline. Why do we interpret the rhinoceros, say, as a solid when it not only lacks any eidolic cues but 
presents strong counter cues? Evidently it is because we know that rhinoceroses are solid objects; ie our interpretation of the linear configuration as occluding bounds derives from prior knowledge. If we did not know what a rhinoceros looked like, obviously we could not identify the figure as such, though we would very likely assume it to be some kind of animal; in fact, we would probably assume it to be some kind of quadruped, in spite of the fact that it has only two legs, because of our prior knowledge that no bipedal animal has its legs so disposed. If, however, we had no knowledge of what an animal looked like or could look like, we would probably see only a meaninglessly meandering enclosed line.

Figure 6 is a greatly abbreviated representation of a mammoth. This may not be too easy to recognize, though in its context of a series of more replete depictions of mammoths in the Rouffignac cave, it is fairly obvious. But again, its recognition as a mammoth is entirely due to knowledge of what a mammoth looks like. Without such knowledge, the representation would appear to be no more than a curvy line without any semantic reference. The figure shows how minimal outline representation can be and still afford recognition, provided it represents some familiar or recognizable object. It is accomplished by the selection of salient and distinctive features.

It should be noted that the kind of reproductions used here and in virtually all publications are rather misleading in that they reduce or eliminate surface 'noise'. Particularly in the case of rock engravings, the competition of natural cracks is sometimes overwhelming. Often an engraved figure simply cannot be extracted from a jumble of lines unless it is properly lit, usually from the side where the engraver's lamp or torch would have originally been placed, and even then it is often necessary for a guide to point out the anatomical components. Everyone who has visited the caves has had this experience, which, with luck, culminates in a moment of sudden recognition when the entire figure miraculously emerges.

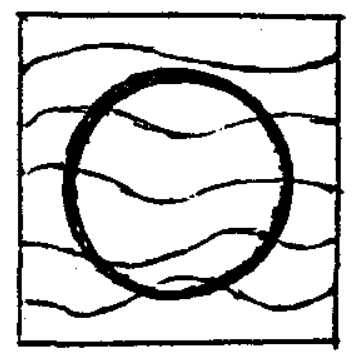

Figure 5. A geometric circle depicted on a background of patterns which continue through the outline figure is interpreted as a hoop, not a disc, sphere, or hole (cf figure 1).

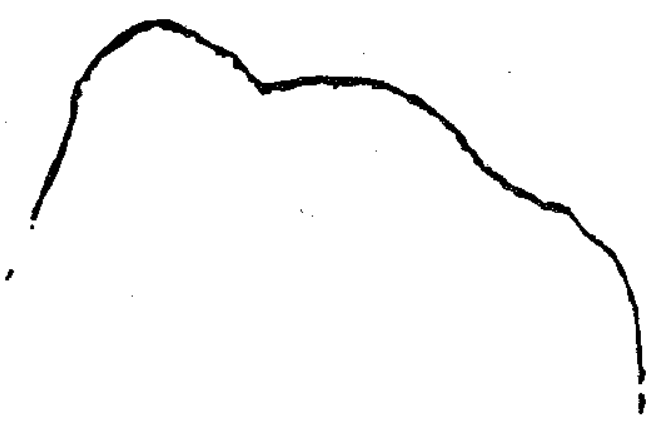

Figure 6. Incomplete representation of a mammoth from the Rouffignac cave. 


\subsection{Feature analysis}

In such instances, it seems evident that visual recognition is a cumulative process, one feature being added to another until a figure begins to be perceived. This suggests a "kind of hypothesis testing game" (Green and Courtis 1966; cf Gregory 1974). As Deregowski puts it, "the observer proceeds by putting forward perceptual hypotheses on the basis of some of the data present and by verifying them against other data available until he reaches a satisfactory solution" (Deregowski 1980, page 118). The "other data" would surely include prior kowledge in the form of "a schema already built up as a result of commerce with the object represented" (Green and Courtis 1966, page 22). One way of accounting for the recognition process posits that schemata or 'norm images' (Arnheim 1969) or 'canonical forms' (Hochberg 1972) are established in memory and that recognition is a process of matching present observation with such generalized images. That the recognition of individual features is part of the process is suggested by the fact that they can normally be described by subjects. So whether object recognition is mainly by differentiation or by pattern construction (Kennedy 1974a), feature analysis is presupposed in either case.

Paleolithic art has its share of ambiguous figures in which certain features can be extracted but do not provide sufficient information for synthesis into a recognizable object (Dodwell 1975). This may be because of projective inaccuracy, but probably more often results from the incompleteness of the depiction and the absence of identifying features. Of greater interest, however, are the many incomplete depictions which can be readily identified despite being only partial representations. Animal heads without bodies are common and are usually easy to recognize. In many cases these are evidently not just unfinished pictures, but contain all the artists intended to represent, as when the surface provides no space for the rest of the figure. Often figures are relatively complete except for the lower body or legs, and very often the lower parts of legs or hooves are missing. Figure 7 shows examples of partial figures: two ibexes, a cow, and three horses. In ail such partial representations it is only the viewer's imagination that completes the figures and thus allows identification to occur. Whatever 'completion' takes place operates at a different level from that of the percept itself, which remains unaltered; it is an interpretation of the percept, indicating "implication processes" rather than "imposition processes" (Kennedy 1971, 1974c).

But does such 'completion' occur? That it does seems to be implied by considerations of reference. If asked what figure $7 \mathrm{a}$ represents, for example, we would normally answer that it shows an ibex or a picture of an ibex, not that it shows a piece of an ibex or a picture of a piece of an ibex. In other words, we assume it is a pars pro toto representation, ie that it refers to a whole animal and not, say, to the remains of a hyena feast. Or again, in figure $7 e$, though we see that parts are missing in the depiction, we do not assume that they are missing in the horse thus represented.

The pars pro toto assumption is well exemplified by the popular interpretation of a series of stag heads in the Lascaux nave (figure 8) as swimming deer, the dark strip of rock beneath serving to represent the stream in which they are immersed. Given the otherwise complete absence of such scenic depictions in Paleolithic art, it is unlikely that this interpretation is valid, but our perceptual preference, so to speak, is not to see these figures as mere disembodied heads, but to complete them imaginatively and in this instance to explain them fictionally.

\subsection{Gestalt processes}

Although 'completion' is an interpretive process, it nevertheless involves perceptual processes as well, in particular Gestalt principles of 'closure' and 'good continuation'. The first refers to the propensity to 'see' closed rather than open forms. Some variant of figure 9 is often used to illustrate this phenomenon, where the normal first 

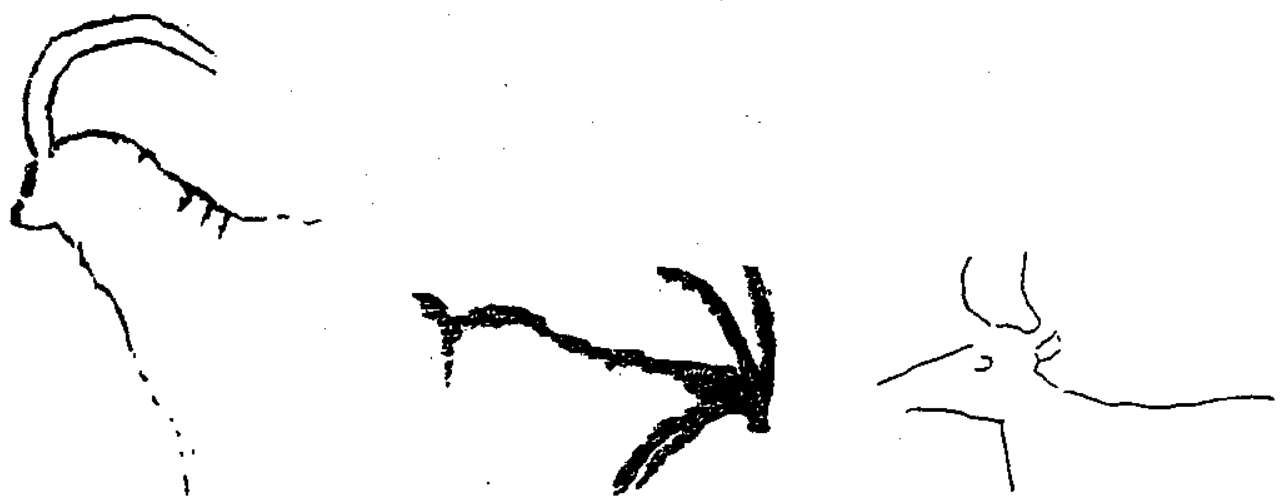

(b)

(c)
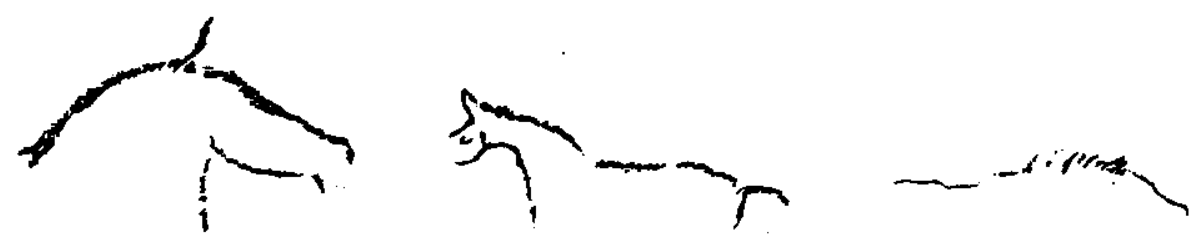

(d)

(e)

(f)

Figure 7. Partial figures of (a) and (b) an ibex, (c) a cow, and $(d),(e)$, and (f) a horse.

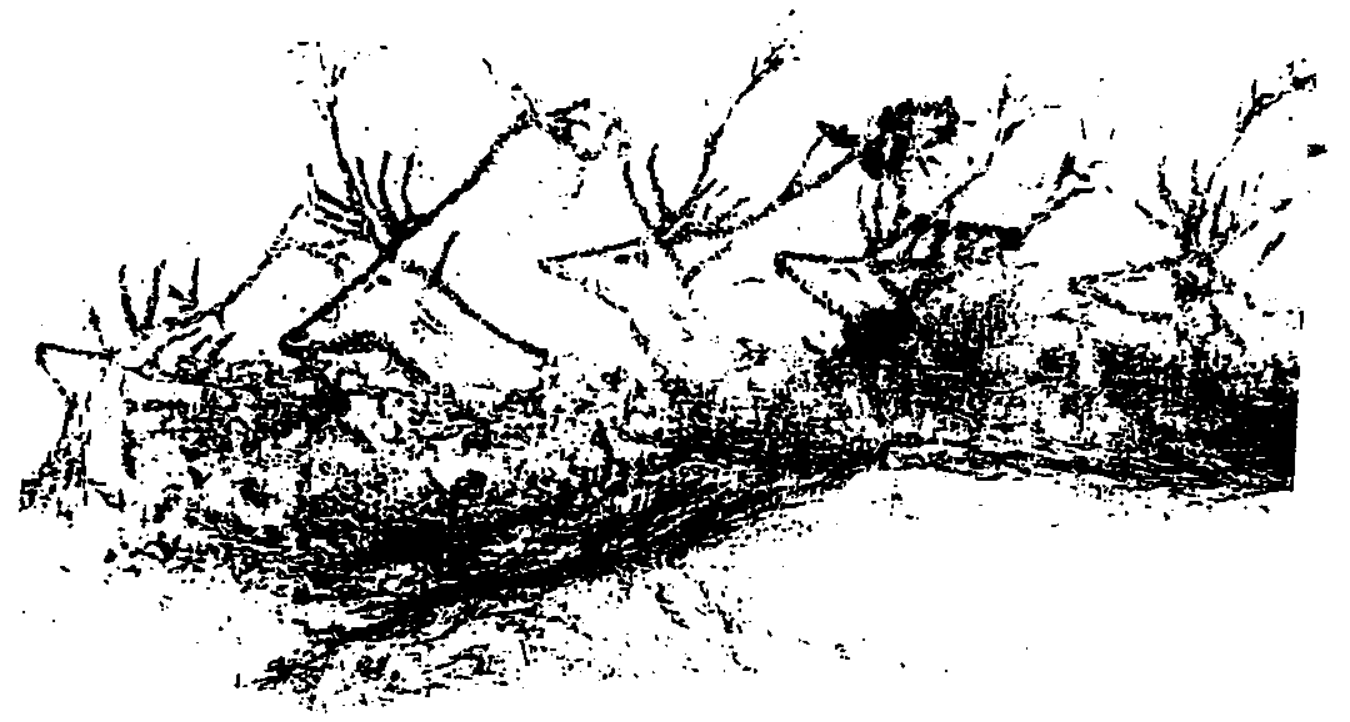

Figure 8. A series of stag heads in the Lascaux cave, popularly interpreted as representing swimming deer.
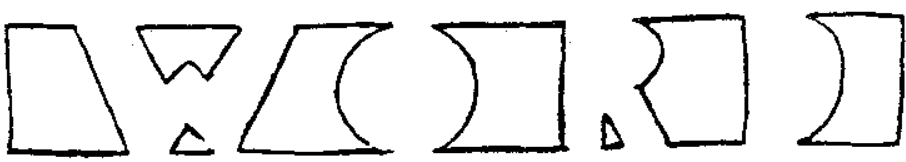

Figure 9. Illustration of the propensity to 'see' closed rather than open forms. 
response is to see some meaningless geometrical shapes before the open forms reveal the word. 'Good continuation' is the propensity to 'see' a line as continuing in one path rather than turning upon itself. In figure 10, for example, we normally interpret the lines as crossed diagonals rather than as tangential angles. In some instances, good continuation effects closure, as in figure 11 where the lines of the angles are normally extended to form an enclosed triangle; this is the most appropriate model for the partial representations of Paleolithic art. However, good continuation is evidently not determined by simple geometric extension; otherwise in figure $7 \mathrm{c}$, for example, the muzzle lines would continue to an unacceptable point, and the back and breast lines would continue ad infinitum. It would seem to be determined, rather, by matching with a mental norm image. Prior knowledge of bovine appearance allows the missing lines to be completed appropriately.

The power of good continuation and the interference of surface noise in the form of natural fissures are both well shown in the common interpretation of the figure at the entrance of Altamira's Gran Sala (figure 12) as a leaping boar when it is almost certainly a bison (Freeman 1987). The traditional misreading (eg García Guinea 1979) is the result of interpreting a pattern of natural fissures as a pig-like snout and as being part of the painted figure-in spite of the fact that the figure has a discernible beard and partially discernible horns, neither of which are characteristic of boars. The crack that looks like the upper boundary of a snout is a 'good continuation' of the painted dorsal line of the animal figure (though the snout line itself is neither painted nor engraved) and is perceptually strong enough to overrule the counterindications of the paint. Freeman has now been able to confirm the bison interpretation by his discovery of a finely engraved outline of the forehead of the bison, where the paint has been lost, that intersects the upper and lower fissures.

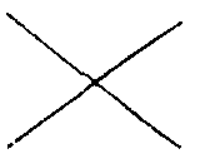

(a)

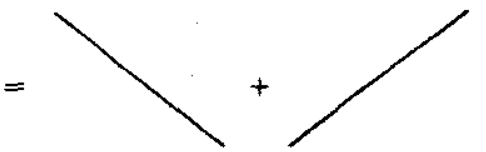

(b) not

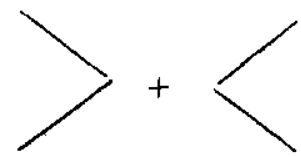

(c)

Figure 10. An example of good continuation: the cross in (a) is normally interpreted as being made up of crossed diagonals (b) rather than tangential angles (c).

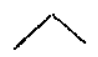

\section{$\angle$}

Figure 11. An example of good continuation resulting in closure: the lines of the angles are usually extended to form an enclosed triangle.

\subsection{Figure 'projection'}

Figure 12 may be used to illustrate another important aspect of Paleolithic art, the frequent use of natural rock features in the creation of the image. This is difficult to show in reproductions, but it is unmistakable in actual viewing, and recurs often. Here it can be seen that the painted dorsal line of the animal figure follows a pattern of natural fissures. Presumably the artist saw in these cracks the shape of a bison's back and was inspired to 'complete' the figure as he did, following the pattern of a norm image, which also obliged the artist to disregard other fissures irrelevant to the image. It is much like a modern viewer pointing to a natural formation and saying, 
"Doesn't this look like the back of a bison?" while tracing with his finger the line observed and then continuing to outline the rest of the figure gesturally. Substitute a burin, manganese crayon, or paint brush for the finger, and an enduring depiction is produced. The 'projection' of familiar images into natural or arbitrary configurations (frosty windowpanes, ink blots, etc) is a basic human propensity which there is no reason to assume did not exist in our remote ancestors. This is not to claim (or disclaim) that the tracing of such projections was the origin of graphic art (Gombrich 1961), but simply that Paleolithic artists often made (literally) imaginative use of natural features, and in doing so, used Gestalt principles of closure and good continuation.

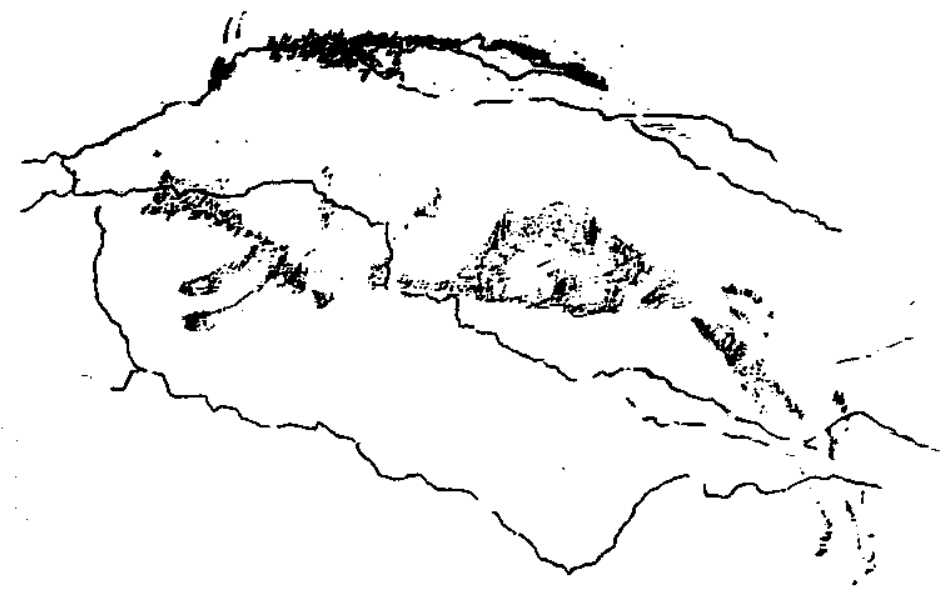

(a)
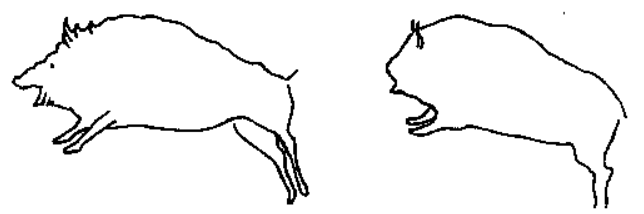

(b)

(c)

Figure 12. The figure (a) at the entrance of Altamira's Gran Sala is commonly interpreted as a boar [b; after Breuil (1979)] rather than, as is more likely, a bison [c; after Freeman (1987)].

\section{Propensity for pictorial perception}

Behind the perception of both imaginative projections and outline drawings there seems to be what Gibson (1951) has called "the pictorial attitude". It is almost as if there were a perceptual imperative to see linear forms as objects wherever possible. When any configuration, especially an enclosed figure, suggests-that is, shares formal features with-a three-dimensional object, it is extremely difficult, if not impossible, to resolve the figure into simple two-dimensional lines. This is evident with many reversal figures such as the Necker cube, which may switch orientation, but can only with the greatest effort, and then only momentarily, be seen as a flat aggregate of triangles, trapezoids, and squares. Even such fairly unlikely shapes as those shown in figure 13 which were used by Gibson (1951) experimentally, were almost always identified as things, rarely as geometrical lines, and never as simply marks on paper. So strong is the propensity to perceive form that, once seen, such figures may even acquire optical effects of luminosity and salience that the displays by themselves cannot account for. And whatever the surface, it tends to resolve itself into foreground and background. 
All of this applies a fortiori to Paleolithic animal art. Outline drawings perceived as animal figures can hardly be perceived as anything else. Even with the minimal, barely suggestive outline of the mammoth shown in figure 6 , once grasped as such, it is virtually impossible to see it as only a curving line; it is a mammoth. The more typical enclosed ontlines are inevitably perceived as figures on a ground, despite their obvious flatness, and as solid despite their obvious transparency. These effects seem to be built into the visual apparatus; there is no reason, then, to suppose that their perception presupposes the learning of pictorial 'conventions' (Goodman 1968; cf Schier 1986).

\subsection{Culture and convention}

Of course, stylistic convention is an indubitable reality of art history-Egyptian art is often cited as exemplary-and may well have existed in Paleolithic art, though this is by no means obvious, but it may be noted that even so highly conventional a tradition as the Egyptian presents no difficulty to observers in identifying figures at the perceptual level. We recognize that this figure is a man, this a woman, that a bird, that a palm tree, etc. We even recognize that a certain figure is a man with the head of a bird. Whatever symbolic value or reference the figure may have is indeed a matter of social convention, but that is quite another matter, involving social cognition rather than visual perception. We may or may not know that the figure represents the god Horus, but what we see is a bird-headed man. It is true that in some art traditions, figures may become stylized beyond recognition by a cultural outsider. In this case, too, the conventions must be learned. But the reason such figures are not recognizable is because they lack sufficient isomorphism with the depicted (or, rather, symbolized) object, which only confirms the perceptual foundations of recognition.

Artistic conventions seem pretty clearly to be secondary, culture-specific developments based on primary, species-specific biological structures and processes depictively reflected in outline drawings. The universality of such drawings argues against any notion of primary conventionalism. On the whole, the issue seems to be something of a red herring, at least in the case of basic line drawings, and is generally rejected by psychologists (eg Deregowski 1989; Hochberg 1978; Kennedy 1974b; Rock 1984).

Nevertheless, there may be certain culturally related skills involved in pictorial perception (Deregowski 1989; Serpell and Deregowski 1980), a possibility raised especially by the occasional failure on the part of modern people who are inexperienced with looking at pictures either to see a depiction at all or to identify it correctly. Such failure is actually very uncommon, so uncommon indeed as to suggest other causes than the lack of inherent pictorial ability. Rather, it "almost certainly reflects the operation of certain inhibitory or distracting processes". Hence "part of the skill of the experienced pictorial perceiver resides in the active suppression of these interfering cues in favour of those required for pictorial perception" (Serpell and Deregowski 1980 , page 159). This is no doubt true in such a case as that reported by Deregowski et al (1972) where African subjects being shown drawings for the first time did not respond to the depictions but rather to the unfamiliar material, that is the paper, by feeling, sniffing, etc. But conversely, where interfering cues are lacking or minimal and so no suppression of them required, presumably no special skill is involved; the inherent ability to comprehend pictures operates freely. Such would be the case, by
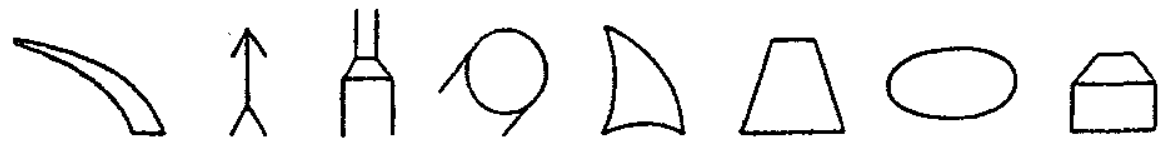

Figure 13. These shapes, used in a study by Gibson (1951), were almost always identified as things rather than as geometrical lines. 
and large, with Paleolithic depiction, where the pictorial support is always the completely familiar material of stone or bone. A great many surfaces are also relatively smooth and devoid of interfering cues. Where there is visual noise from natural fissures, as in the case of many engravings, resolving ambiguities would seem to follow basic Gestalt processes, rather than depend on acquired skills. Paintings have even fewer interfering cues because the artificial nature of paint usually precludes confusion with anything in the natural substrate. As far as Paleolithic depiction in general is concerned, then, there would seem to be no reason to regard "pictorial perception as a culturally specific skill" (Serpell and Deregowski 1980, page 175). Culture would be relevant to pictorial perception only in the sense that recognition of the object depicted would depend on familiarity with the object, which would of course be determined by the life experiences afforded by a particular culture.

\subsection{Salient features}

The recognition process accommodates an extreme latitude of representation, from a single line to a complete form, and can also accommodate considerable distortion, or "affine transformations" (Hagen 1986), and degradation. The figures shown in figure 14, for example, are hardly more than crude caricatures, yet are readily recognizable as a bison and a rhinoceros. Probably the essential element in such recognition is feature saliency. Once salient features are adequately represented, the rest of the image falls into place. But what are salient features? Tversky (1977) has defined the salience of a feature as being either "intensive" or "diagnostic", the latter referring to "the classificatory significance of features" (Tversky 1977, page 342). In the context of Paleolithic art, a diagnostic feature is one which is sufficiently distinctive to classify an animal species, such as the unmistakable horns of the rhinoceros. This is sound, but perhaps should be modified to include effects from habitual perception. The most diagnostic feature of a mammoth, for example, would seem to be its unique tusks, but, as figure 6 shows, its dorsal line could be used as well, a feature presumably derived from a distant view of the animal in high grass, indicating that habitual perception may also play a part in feature saliency.

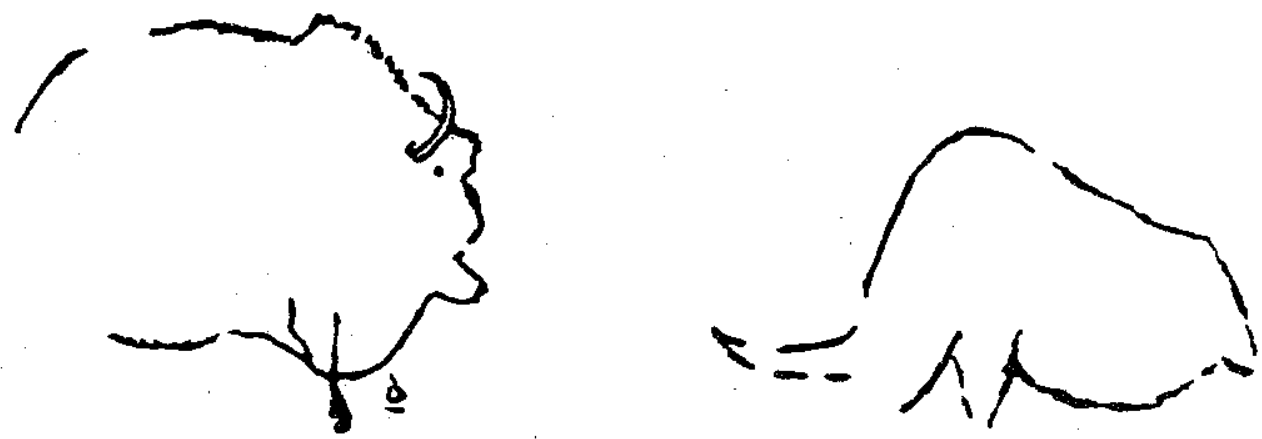

Figure 14. Crude figures readily recognizable as a bison and a rhinoceros.

\section{Conclusions}

At the beginning of this essay I raised the question of why Paleolithic art looks the way it does. It appears that perceptual psychology can shed light on the subject. It may also be that the study of the first pictures can, reciprocally, confirm or support some of the findings of psychology. Some of the more important characteristics of primal depiction discussed here are outline style, partial representation, and profile perspective. These may be profitably approached by, respectively, studies of Gestalt principles and line surrogacy, feature analysis, and canonical bias in representation. 
It is of some interest that the earliest two-dimensional depictions known should be outline drawings, either engraved or painted. They exhibit first of all what appears to be the most fundamental connection between perception and graphic representation, namely line surrogacy, which works, it has been argued, because it engages the same perceptual faculties, and in the same way, as does three-dimensional viewing. "Outlines will serve as surrogates for objects' edges because they share the same receptor mechanisms that are sensitive to luminance differences, and because most objects' edges and corners are marked by an abrupt luminance change that remains detectable in peripheral vision where other information, more powerful but more dependent on detail, fails" (Hochberg 1978, page 240; cf Zusne 1970, page 17). If this position is correct, even approximately, it would seem virtually predictable that graphic depiction could have begun only in the outline form it shows. It is predictable too, perhaps, that if any depictions earlier than those we now have should turn up in the archeological record, (a pre-prehistory of depiction has often been suggested) they will have the same outline characteristics.

Outlines are of forms. Gestalt psychology has been most concerned with analyzing the processes of form searching, and some of its basic observations have endured very well. Figure-ground distinction, good continuation, and closure are useful principles in understanding both how depictions are made and how they are recognized. Feature analysis seems to be presupposed by the numerous partial representations in Paleolithic art, both in their production and in their recognition. It is also consistent with Biederman's general theory of image understanding and his "principle of componential recovery", that states that "If the components in their specified arrangement can be readily identified, object identification will be fast and accurate" (Biederman 1987, page 139).

Distinctive features, especially distinguishing or diagnostic features, also have a role in 'canonical representation', which "depicts the object in an orientation which contains the important structural features necessary for recognition" (Davis 1985, page 202). Freeman and Janikoun (1972), for example, found that most young children when asked to draw a cup in front of them that was turned so that the handle was not visible to them nevertheless included the handle in their drawing, thus demonstrating a canonical bias towards the distinguishing feature. Such features, with the same kind of information load, are characteristic of Paleolithic art.

Characteristic, too, is canonical perspective: the angle of view "that reveals the most information of greatest salience" about the object, salience being defined as concerning "the perceiver's familiarity with the revealed aspects and their importance within his/her knowledge about objects" (Palmer et al 1981, page 147). In their experiments, in which subjective judgments of 'goodness' of perspective, were elicited, Palmer et al (1981) included only one animal figure, a model of a horse, presented in twelve different perspectives; the two highest rated views were a slightly angled profile and a full profile, corresponding almost perfectly to the profile representation so ubiquitous in Paleolithic depiction, leaving little doubt that the artists strongly favored canonical perspective and its potentially maximal "subjective information content".

All the perceptual processes are firmly rooted in evolution. The visual system evolved as it did because the human species evolved as it did in response to particular ecological and biological circumstances, in which the ability to distinguish figure and ground and to correlate visual contours with solid objects was an adaptation probably necessary for survival. To the extent that depictions may induce the same neurophysiological responses as the occluding bounds of solid objects, it is not surprising that the recognition of outline pictures should require no more (or less) learning than that required for the recognition of objects. It is perhaps more surprising that the obvious 
flatness, transparency, lack of motion parallax, simplification, and distortions of pictorial representations should be suppressed or overridden in pictorial perception; but the fact is that they are. As Deregowski (1989) says, "the eye is very tolerant" (page 103).

The ability to make or comprehend pictures has no self-evident adaptive value. Although cultural evolution eventually made it an extremely valuable, if not quite indispensable, asset, it is not easy to see how depiction would have conferred any biological advantage on early humans. Picture perception, like many other aspects of human culture, seems to be a spillover phenomenon, the accidental transfer of an ecologically evolved, adaptive biological trait to a purely cultural modality. The importance of form in visual perception-form that in some cases is determined not just primarily but entirely by occluding edges-has a readily understandable evolutionary history. It is this fundamental feature of perception that is transferred to line surrogacy, which is in turn the foundation of all graphic art.

Acknowledgements. I would like to thank Professors Jan Deregowski and Irvin Rock for helpful advice and encouragement. I am particularly grateful to Professor John M Kennedy for his careful reading of the manuscript and for numerous useful criticisms.

\section{References}

Arnheim R, 1969 Visual Thinking (Berkeley: University of California Press)

Bahn P G, Vertut J, 1988 Images of the Ice Age (New York: Facts on File)

Biederman I, 1987 "Recognition-by-components: A theory of human image understanding" Psychological Review 94 115-147

Biederman 1, Ju G, 1988 "Surface versus edge-based determinants of visual recognition" Cognitive Psychology $2038 \sim 64$

Breuil H, 1952/1979 Four Centuries of Cave Art; English translation by M E Boyle (New York: Hacker Art Books, 1979) of Quatre Cent Siecles d'Art Pariétal (Montignac: Centre d'Études et de Documentation Préhistoriques, 1952)

Cabe PA, 1980 "Picture perception in nonhuman subjects" in The Perception of Pictures volume 2, Ed. M A Hagen (New York: Academic Press) pp 305-343

Davis A M, 1985 "The canonical bias: Young children's drawings of familiar objects" in Visual Order Eds N H Freeman, M V Cox 〈Cambridge: Cambridge University Press) pp 202-213

Deregowski J B, 1976 "On seeing a picture for the first time" Leonardo 9 19-23

Deregowski J B, 1980 Illusions, Patterns, and Pictures (London: Academic Press)

Deregowski J B, 1989 "Real space and represented space: Cross-cultural perspectives" Behavioral and Brain Sciences $1251-119$

Deregowski J B, 1990 "On two distinct and quintessential kinds of pictorial representation" in Ecological Perception Research, Visual Communication, and Aesthetics Ed. K Landwehr (Berlin: Springer) pp 29-42

Deregowski J B, Muldrow E S, Muldrow W F, 1972 "Pictorial recognition in a remote Ethiopian population" Perception $1417-425$

Dodwell P C, 1975 "Pattern and object perception" in Handbook of Perception volume 5, Eds E C Carterette, M P Friedman (New York: Academic Press) pp 261-299

Forge A, 1970 "Learning to see in New Guinea" in Socialization: The Approach from Social Anthropology Ed. P Mayer (New York: Tavistock) pp 269-291

Freeman L.G, 1987 Altamira Revisited (Chicago/Santander: Institute for Prehistoric Investigations)

Freeman N H, Janikoun R, 1972 "Intellectual realism in children's drawings of a familiar object with distinctive features" Child Development $431116-1121$

García Guinea M A, 1979 Altamira and Other Cantabrian Caves translated by N Polak (Madrid: Silex)

Gibson J J, 1951 "What is a form?" Psychological Review 58 403-412

Gombrich E H, 1961 Art and Illusion (Princeton, NI: Princeton University Press)

Goodman N, 1968 The Languages of Art (Indianapolis, IN: Bobbs-Merrill)

Green R T, Courtis M C, 1966 "Information theory and figure perception: The metaphor that failed" Acta Psychologica $2512-36$

Gregory R J, 1974 "Choosing a paradigm for perception" in Handbook of Perception volume 1, Eds E C Carterette, M P Friedman(London: Academic Press) pp 255-283 
Haber R N, Hershenson M, 1980 The Psychology of Visual Perception (New York: Holt, Rinehart and Winston)

Hagen M A, 1986 Varieties of Realism (Cambridge: Cambridge University Press)

Hochberg J, 1972 "The representation of things and people" in Art, Perception and Reality Eds E H Gombrich, J Hochberg, M Black (Baltimore, MD: The Johns Hopkins University Press) pp 47-94

Hochberg A, 1978 "Art and perception" in Handbook of Perception volume 10, Eds E C Carterette, M P Friedman (London: Academic Press) pp 225 - 258

Hochberg J, Brooks V, 1962 "Pictorial recognition as an unlearned ability: A study of one child's performance" American Journal of Psychology 75 624-628

Hudson W, 1960 "Pictorial depth perception in subcultural groups in Africa" Journal of Social Psychology 52 183-208

Kennedy J M, 1971 "Incomplete pictures and detection of features" Journal of Structural Learning $171-73$

Kennedy J M, 1974a "Is gradual recognition of pictures by Ethiopian subjects a differentiation process or a pattern construction process?" Perception 3 29-31

Kennedy J M, 1974b A Psychology of Picture Perception (San Francisco: Jossey-Bass)

Kennedy J M, 1974c "Perception, pictures, and the etcetera principle" in Perception Eds R B MacLeod, H L Pick JI (Ithaca, NY: Cornell University Press) pp 209-226

Kennedy J M, Ross A S, 1975 "Outline picture perception by the Songe of Papua" Perception 4 $391-406$

Kennedy J M, Silver J, 1974 "The surrogate functions of lines in visual perception: Evidence from antipodal rock and cave artwork sources" Perception 3 313-322

Lorblanchet M, 1977 "From naturalism to abstraction in European prehistoric rock art" in Form in Indigenous Art Ed P J Ucko (London: Gerald Duckworth) pp 44-56

Leroi-Gourhan A, 1967 Treasures of Prehistoric Art translated by N Guterman (New York: Harry N Abrams)

Leroi-Gourhan A, 1982 The Dawn of European Art translated by S Champion (Cambridge: Cambridge University Press)

Mundy-Castle A C, 1966 "Pictorial depth perception in Ghanajan children" International Journal of Psychology $1289-300$

Palmer S, Rosch E, Chase P, 1981 "Canonical perspective and the perception of objects" in Attention and Performance Volume IX, Eds $\mathrm{J}$ Long, A Baddeley (Hillside, NJ: Lawrence Erlbaum) pp 135-151

Rock I, 1984 Perception (New York: Scientific American Library)

Sandars N K, 1985 Prehistoric Art in Europe (Harmondsworth, Middlesex: Penguin Books)

Schier F, 1986 Deeper into Pictures (Cambridge: Cambridge University Press)

Segall M H, Campbell D T, Herskovits M J, 1966 The Influence of Culture on Visual Perception (Indianapolis, IN: Bobbs-Merrill)

Serpell R, Deregowski J B, 1980 "The skill of pictorial perception: An intepretation of crosscultural evidence" International Journal of Psychology 15 145-180

Tversky A, 1977 "Features of similarity" Psychology Review 84 327-350

Ucko P J, Rosenfeld A, 1967 Paleolithic Cave Art (New York: McGraw-Hill)

Zusne L, 1970 Visual Perception of Form (New York: Academic Press) 